\title{
THE DYNAMICAL EVOLUTION OF A CLUMPY MEDIUM
}

\author{
H.W. Yorke, R. Kunze, R. Spurzem \\ Institut für Astronomie und Astrophysik \\ der Universität Würzburg, Am Hubland, \\ D-8700 Würzburg, Federal Republic Germany
}

\begin{abstract}
We consider the evolution of a multicomponent medium: stars and gas clouds embedded in an ambient gaseous medium. The stars and gas clouds are treated stellar dynamically, using a velocity moment expansion of the Boltzmann equation. The basic equations and interaction terms are discussed. Examples of 2-D numerical calculations are presented (see also Kunze et al., this meeting).
\end{abstract}

\subsection{Introduction}

The evolution of interstellar gas is often studied by numerical solution of the gas dynamic equations. Due to memory and CPU time, the discretization of these gas dynamical equations is restricted to $\lesssim 10^{\mathrm{s}}$ grid cells in an explicit calculation. This means that for a 2-D calculation the grid size is $\Delta r \gtrsim 10^{-3.5} \mathrm{~L}$ where $L$ is the overall length scale of the problem. When considering the nucleus of a galaxy, for instance, $L \sim 1 \mathrm{kpc}$ and $\triangle r \gtrsim 3 \mathrm{pc}$ (for implicit calculations it is possible to use varying grid scales over several orders of magnitude). Considering a volume of the order $(\Delta r)^{3}$, the number of particles is sufficiently large to be treated statistically and the velocity distributions generally sufficiently Maxwellian - except in the case of shocks - that the gas dynamical equations apply.

If, however, the interstellar gas is clumpy on scales of the order of a few times the grid size or smaller, then the discretized gas dynamical equations are no longer valid. The numerical resolution of gas dynamical codes is insufficient to follow the evolution of the interstellar gas on both large and small length scales. It is important to realize that this may significantly affect the overall evolution. In the following we describe a numerical procedure for treating such a clumpy medium.

\subsection{The gas model for stellar systems}

Consider the Boltzmann equation (see e.g. Binney and Tremaine 1987)

$$
\frac{\partial f}{\partial t}+\left(\mathbf{u} \cdot \nabla_{\mathrm{r}}\right) f+\left(\mathbf{a} \cdot \nabla_{\mathrm{u}}\right) f=\left(\frac{\delta f}{\delta t}\right)_{\mathrm{c}},
$$

where $f=f(\mathbf{r}, \mathbf{u}, t)$ is the one-particle distribution function (phase space density of particles), $\mathrm{a}=d \mathrm{u} / d t$ is the acceleration, and $(\delta f / \delta t)_{c}$ is the collision term, describing the sudden jump of particles from one part of phase space to another. We define velocity moments of the distribution function by $\langle\ln m\rangle=\int u_{x}^{l} u_{y}^{n} u_{x}^{m} f d u_{x} d u_{y} d u_{x}$. Multiplying the Boltzmann equation by the velocity components to various powers and integration over velocity space yields for e.g. Cartesian coordinates the moment equations 


$$
\begin{aligned}
& \frac{\partial}{\partial t}\langle 000\rangle+\frac{\partial}{\partial x}\langle 100\rangle+\frac{\partial}{\partial y}\langle 010\rangle+\frac{\partial}{\partial z}\langle 001\rangle=\cdots \\
& \frac{\partial}{\partial t}\langle 100\rangle+\frac{\partial}{\partial x}\langle 200\rangle+\frac{\partial}{\partial y}\langle 110\rangle+\frac{\partial}{\partial z}\langle 101\rangle+\cdots
\end{aligned}
$$

By defining $\rho_{*}=\langle 000\rangle, \rho_{*} v_{x}=\langle 100\rangle$, etc. the first equation in this series resembles the equation of continuity

$$
\frac{\partial \rho_{*}}{\partial t}+\nabla \cdot\left(\rho_{*} v\right)=\sum_{i}\left(\frac{\delta \rho_{*}}{\delta t}\right)_{i}
$$

In order to be computer-solvable, this sequence of moment equations of ascending order must be truncated at some level and complemented by a closure relation, which relates the moments of the maximum order to moments of lower order. This set of equations resembles those of gas dynamics, except for the effects of anisotropy, possible collisional terms and the particular choice of the closure relation. Such a system of moment equations has been successfully applied to interesting stellar dynamical problems (Bettwieser and Spurzem 1986) and compares well with other methods of calculating stellar dynamics (cf. e.g. Cohn 1985, Heggie 1985, Bettwieser and Sugimoto 1985).

\section{The physical description of a multi-component medium}

We interprete a "clumpy" medium as a system of "clouds" moving in a system of point masses (stars) and an ambient intercloud medium (ICM). Considering the equations of the ICM component our treatment is equivalent to the work of Cowie et al. (1981); their method is extended here by the assumption that a separate Boltzmann equation applies for every component. In addition to the usual gas dynamical equations for the ICM this yields a set of moment equations up to second order (see section 2 and also Scalo and Struck-Marcell 1984). Moreover, our code includes moment equations for the stellar component (cf. Langbein et al. 1989), but for the present investigation it suffices to consider the stellar component as stationary. "Phase transitions" and interactions among the components (drag forces on clouds moving relative to the ICM) as depicted in Fig. 1 are considered similarly as in Cowie et al. (1981). The decrease of cloud kinetic energy by cloud-cloud collisions is also taken into account.

We calculate the evolution of supernova remnants expanding into a medium in which a fraction of the total mass is in form of clouds. Depending on its temperature, the ICM can depict either the hot $\left(T \sim 10^{6} \mathrm{~K}\right)$ or warm $\left(T \sim 10^{4} \mathrm{~K}\right)$ component of the ISM. Cooling of the gaseous component is considered according to the cooling function of Raymond et al. (1976), modified in our 2-D calculations by the assumption that any attempt to cool below $10^{4} \mathrm{~K}$ only results in an increased rate of cloud condensation (process 3 in Fig. 1). This process is only important in the dense shell of the remnant. For simplicity we only consider clouds of a single mass $\left(m_{2}=10 \mathrm{M}_{\odot}\right)$ and a single radius $(a=1.7 \mathrm{pc})$. We shall give a more complete description our numerical model elsewhere.

\subsection{The numerical model}

\subsection{Basic assumptions}

The gas dynamic equations which describe the ICM simultaneously with the moment equations for the cloud system are solved with an explicit 2-D second-order scheme 


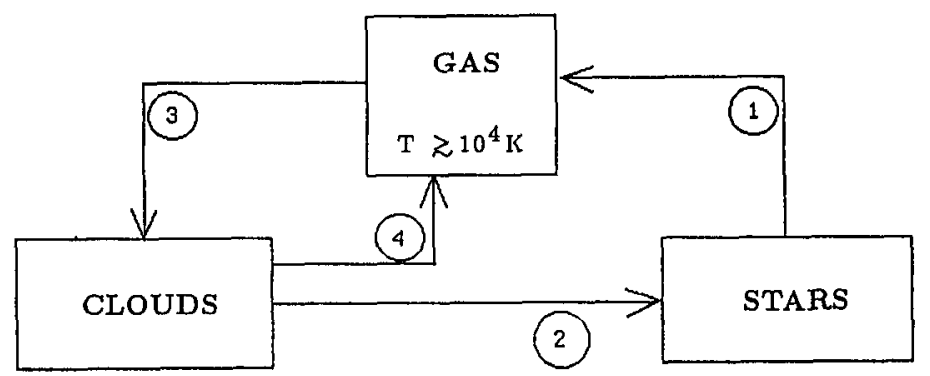

Fig. 1. The three components considered in the present investigation and some of their associated phase transitions and interactions: 1) mass loss from aging and dying stars; 2) star formation; 3) condensation of clouds; 4) destruction of clouds by champagne flows, evaporation (heat conduction at cloud/gas interface).

(see Różyczka, 1985, for more details). The calculations are performed on a cylindrical grid with $100 \times 50$ uniformly distributed cells with a resolution of $5 \mathrm{pc}$ in both Z- and $R$-directions. Axial symmetry with respect to the $Z$-axis and mirror symmetry with respect to the $Z=0$ plane are assumed.

Magnetic fields and heat conduction in the ICM gas are presently neglected. Cooling of the intercloud gas is included although we do not allow the gas to cool below $10^{4} \mathrm{~K}$. The intercloud gas is assumed to be fully ionized while the gas inside the clouds is completely neutral. Gravity is neglected except for case 2 where a gravitational potential was chosen in order to hold the stratified medium in hydrostatic equilibrium.

We model the simultaneous explosions of 10 supernovae (an "OB association") by the sudden release of thermal energy and density within a small volume. Per supernova $10 \mathrm{M}_{\odot}$ of mass loss and $10^{51} \mathrm{erg}$ of kinetic energy are liberated. Mass and energy are smeared out over the initial volume of the remnant to give the equivalent amounts of mass and energy density. In case 2 a stratified medium is chosen to account for the nonuniformity of the galactic disk. The spatial gas and cloud density are distributed like a Gaussian with scale heights of $200 \mathrm{pc}$ and $140 \mathrm{pc}$, respectively.

\subsection{Results}

case 1. 10 supernovae with a total energy of $10^{52} \mathrm{erg}$ explode into a medium with an ICM density of $\rho_{1}=1.0310^{-25} \mathrm{~g} \mathrm{~cm}^{-3}$ and a smeared out cloud density of $\rho_{2}=$ $1.0210^{-24} \mathrm{~g} \mathrm{~cm}^{-3}$ in a considered volume of $590 \times 250 \mathrm{pc}$.

We compare one calculation without any interaction processes and phase transitions (\#1) with another including these effects (\#2). The results of calculation \#1 show qualitative agreement with other 2-D calculations (e.g. Bodenheimer et al., 1984; Tenorio-Tagle et al., 1987). In calculation \#2 the evolution was qualitatively similar to \#1. The ICM density interior to the remnant, however, grew up to a value about four times larger and the temperature was lowered by the same factor compared to calculation \#1. When the material of the expanding shell has cooled down, its temperature and density again resembled more closely the situation yielded with calculation \#1. In Fig. 2 the density profiles of the ICM and the clouds together with their velocity fields are displayed for the last model of \#2. A comparison of the evolution of the remnants' radii (Fig.3) shows that the remnant in calculation \#2 evolved to a larger radius.

Evaporative mass loss of the clouds is the most effective process during early phases of the remnant's evolution in calculation \#2 caused by the very high temperature of the hot interior. The mean cloud density $\rho_{2}$ falls off there by about one order of magnitude. 
Drag between clouds and ICM and the acceleration of clouds by the pressure gradients across the shock front add approximately a same amount of momentum to the clouds, which achieved a maximum bulk velocity of about $10 \mathrm{~km} \mathrm{~s}^{-1}$ outwards near the remnant's outer boundary.

The smallest effect has been seen by collisions between clouds. During the evolution the velocity dispersion of the clouds changed by only a few percent.

A complementary 1-D implicit calculation has been performed in spherical symmetry for comparison. It is capable for taking into account clouds of different mass simultaneously. Preliminary results show the same basic features of the remnant's evolution as in the 2-D calculations. A central cloud-free zone is established by evaporation and the acceleration of clouds outwards to $\sim 10 \mathrm{~km} \mathrm{~s}^{-1}$. The more massive clouds are less affected by this processes than the lighter ones.

case 2. With the same free parameters as in case 1 we now let 10 supernovae explode at $z=0$ into a stratified medium. Again we compare the evolution of the remnant with and without interaction terms between the cloud and ICM components. In both calculations the remnant became clearly aspherical after an evolution of $\sim 10^{6} \mathrm{yr}$. The shell cooled down to $\approx 10^{4} \mathrm{~K}$ even in the parts of the remnant perpendicular to the plane. Here, cloud condensation is enhanced.

\subsection{Discussion and conclusions}

In the presence of a "clumpy" medium consisting of "H I clouds" and a warm $\left(\sim 10^{4} \mathrm{~K}\right)$ intercloud medium the evolution of a supernova remnant seems not to be greatly affected. Substantial cloud evaporation takes place only in the early evolutionary phases of the evolution. A relatively large amount of matter is added to the ICM. Due to the evaporation of clouds the remnant does not become as strongly rarefied as in

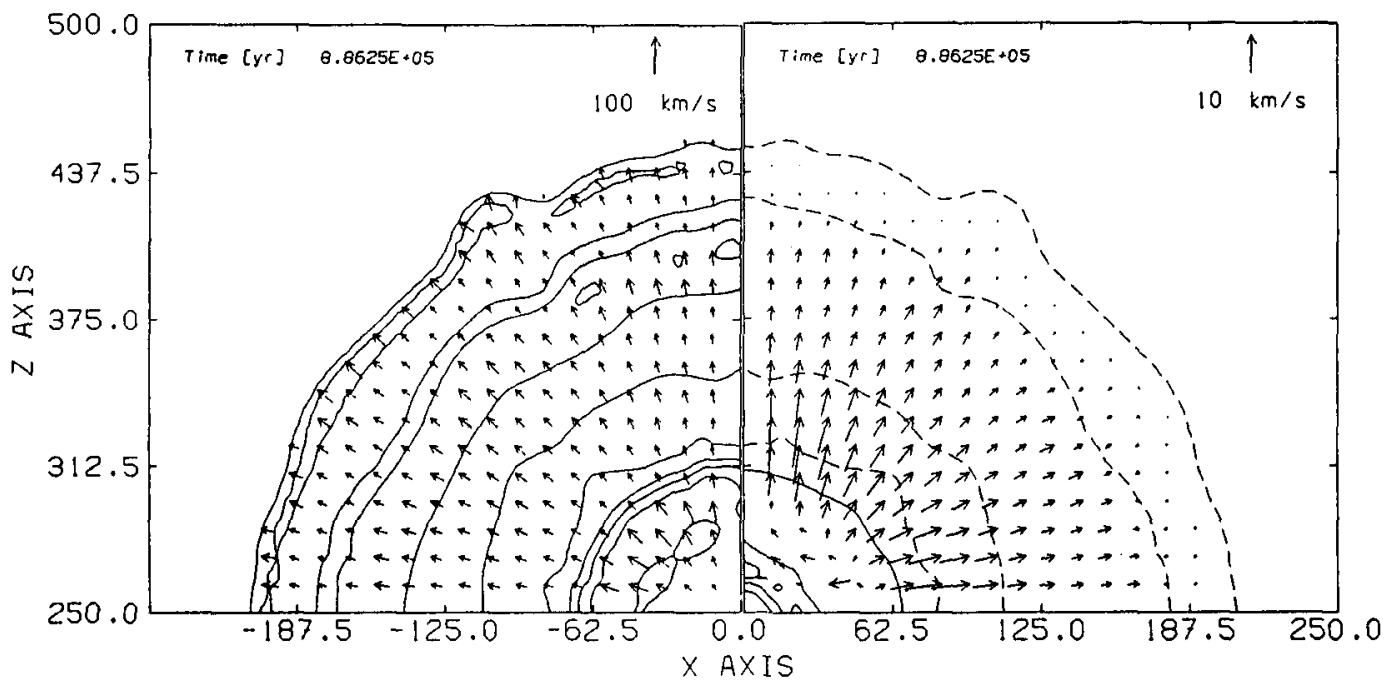

Fig. 2. The supernova remnant of the last model from calculation \#2 of case 1 . Lines of constant ICM density are plotted in a logarithmic scale of $\Delta \log \rho_{1}=0.2$. For some contours the value of $\log \rho_{1}$ (in $\left.\mathrm{g} \mathrm{cm}^{-3}\right)$ is given explicitly. In the second part of the figure isodensities of the cloud component are displayed. Here it is $\triangle \log \rho_{2}=0.05$. For comparison one ICM density contour is added (dashed line). 


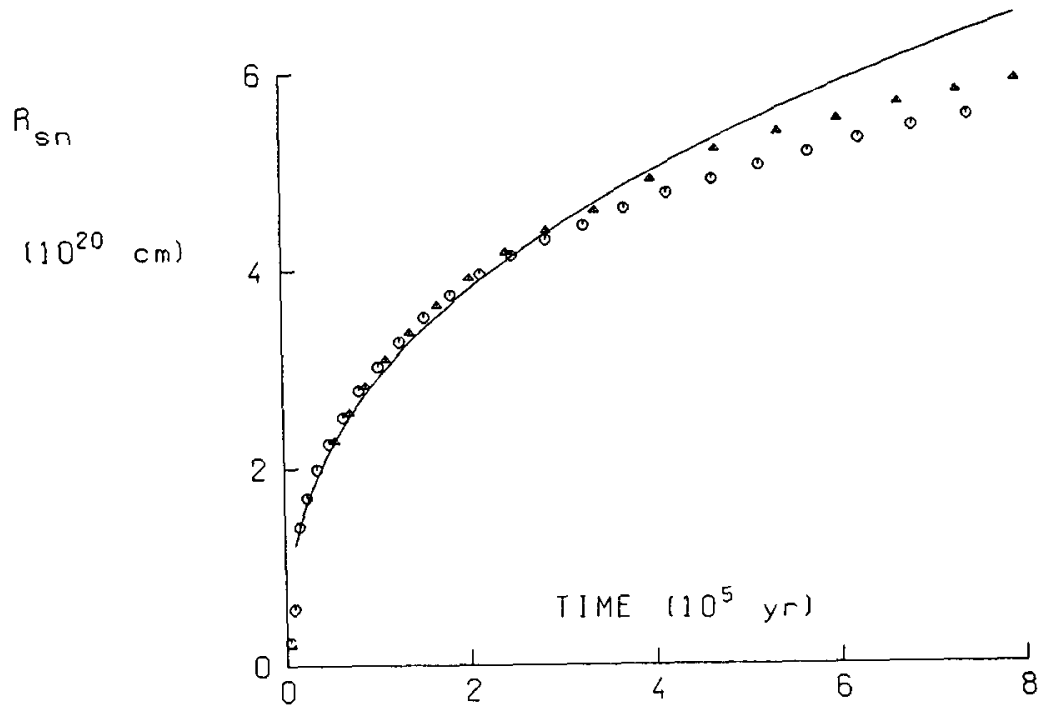

Fig. 3. Evolution of a supernova remnant's radius for case 1. Circles refer to calculation \#1, triangles to \#2. The straight line is the Sedov solution.

the case without clouds; density contrasts interior to the remnant are smeared out. Higher ICM densities inside the remnant lead to lower gas temperatures. Since multiple supernova explosions are often taken into account as a heating source of the galactic halo gas (see eg. Tomisaka and Ikeuchi, 1986; Mac Low et al. 1989), this may affect the heating rate of the galactic halo gas. Further investigation is necessary in order to clarify this point.

The clouds near the outer shell of the remnant are accelerated up to velocities of about $10 \mathrm{~km} \mathrm{~s}^{-1}$ outwards. Consecutive supernova explosions may lead to a further acceleration of the clouds and thus enhance their overall velocity dispersion.

Acknowledgements We acknowledge support by the DFG under grants Yo 5/5-1 and Yo 5/5-2. The calculations have been performed on a VAX 8810 of the "Rechenzentrum der Universität Würzburg".

\section{References}

Bettwieser, E. and Sugimoto, D.: 1985, Monthly Notices Roy. Astron. Soc. 212, 189

Bettwieser, E. and Spurzem, R.: 1986, Astron. Astrophys, 161, 102

Binney, J. and Tremaine, S.: 1987, Galactic Dynamics, Princton Series in Astrophysics

Bodenheimer, P., Yorke, H.W. and Tenorio-Tagle, G.: 1984, Astron. Astrophys. 138, 215

Cohn, H.: 1985, in "Dynamics of star clusters", IA U Symp. 113, eds. J. Goodman, P. Hut, Dordrecht, Reidel, p. 161

Cowie, L.L. and McKee, C.F.: 1977, Astrophys. J. 211, 135

Cowie, L.L., McKee, C.F. and Ostriker, J.P.: 1981, Astrophys. J. 247, 908 
Heggie, D.C.: 1985, in "Dynamics of star clusters", IAU Symp. 113, eds. J. Goodman, P. Hut, Dordrecht, Reidel, p. 139

Kunze, R., Yorke, H.W., Spurzem, R.: 1989, this meeting

Langbein, T., Spurzem, R., Fricke, K.J. and Yorke, H.W.: 1989, submitted to Astron. Astrophys.

Mac Low, M.-M., McCray, R. and Norman, M.L.: 1989, Astrophys. J. 337, 141

Raymond, J.C., Cox, D.P. and Smith, B.W.: 1976, Astrophys. J. 204, 290

Różyczka, M.: 1985, Astron. Astrophys. 143, 59

Scalo, J.M. and Struck-Marcell, C.: 1984, Astrophys. J. 276, 78

Tenorio-Tagle, G., Bodenheimer, P. and Rożyczka, M.: 1987, Astron. Astrophys. 182, 120

Tomisaka, K. and Ikeuchi, S.: 1986, Publ. Astron. Soc. Japan 38, 697

Discussion:

VÖLK: You have plotted the radii of the SNRs vs. time in a linear form. Is there a Sedov phase reconcilable in the figure and if so, at what times does it occur with and without clumps?

YORKE: We find the classical phases of SNR evolution in sequences calculated with and without clumpiness. For the particular set of parameters we choose for our test calculations of an explosion in a homogeneous (or homogeneously clumpy) medium there was little modification. The main differences was an abscense of very low density gas in the SNR interior. Evaporating clumps provided a source of gas here. Also, in a radius versus time diagram, the clumpy calculations got a little further.

PECKER: How do you parametrize the clumpiness? How is its characteristic size defined?

YORKE: We have treated "clumpiness" by considering the evolution of a two component medium. There is a "cloud" component which we treat stellar dynamically and a "gaseous" component which is treated by solving the gas dynamical equations. The interaction terms, of course, implicity contain our cloud model. For this we have to specify typical cloud sizes and internal cloud densities. For one series of calculations we specified the mass of the clouds and their internal temperature. The sizes and internal densities were calculated by assuming presure equilibrium with the gaseous component.

DYSON: I find it hard to understand how putting in clumps and enhancing radiative cooling makes a supernova travel further.

YORKE: By injecting material (evaporating clumps) into the SNR at an early evolutionary phase, when the remmant is still adiabatic, we have enhanced the momentum of the expanding material at the cost of having the radiative phase begin somewhat earlier. The net result for the particular set of parameters we used was that the calculation with cumps got a little further after the snow-plough phase began. Unfortunatelly, I cannot say whether this effect will continue to occur when we begin varying the parameters or whether this is a singular result. We need to do a parameter study. 